\title{
Detection of Rogue Certificates from Trusted Certificate Authorities Using Deep Neural Networks
}

ZHENG DONG, Microsoft Corporation

KEVIN KANE, Microsoft Research

L. JEAN CAMP, Indiana University Bloomington

\begin{abstract}
Rogue certificates are valid certificates issued by a legitimate certificate authority (CA) that are nonetheless untrustworthy; yet trusted by web browsers and users. With the current public key infrastructure, there exists a window of vulnerability between the time a rogue certificate is issued and when it is detected. Rogue certificates from recent compromises have been trusted for as long as weeks before detection and revocation. Previous proposals to close this window of vulnerability require changes in the infrastructure, Internet protocols, or end user experience. We present a method for detecting rogue certificates from trusted CAs developed from a large and timely collection of certificates. This method automates classification by building machine-learning models with Deep Neural Networks (DNN). Despite the scarcity of rogue instances in the dataset, DNN produced a classification method that is proven both in simulation and in the July 2014 compromise of the India CCA. We report the details of the classification method and illustrate that it is repeatable, such as with datasets obtained from crawling. We describe the classification performance under our current research deployment.
\end{abstract}

Categories and Subject Descriptors: K.6.5 [Security and Protection]: Unauthorized Access

General Terms: Security, Design

Additional Key Words and Phrases: Certificates, machine learning

ACM Reference Format:

Zheng Dong, Kevin Kane, and L. Jean Camp. 2016. Detection of rogue certificates from trusted certificate authorities using deep neural networks. ACM Trans. Priv. Secur. 19, 2, Article 5 (September 2016), 31 pages. DOI: http://dx.doi.org/10.1145/2975591

\section{INTRODUCTION}

The reliability of the public key infrastructure (PKI) ecosystem depends on the trustworthiness of the certificate authority (CAs). With the root certificates shipped to the end users as part of the installation of the web browser or operating system, most web browsers make the following two implicit assumptions.

Research was sponsored by the Army Research Laboratory and was accomplished in part under Cooperative Agreement Number W911NF-13-2-0045 (ARL Cyber Security CRA). This material is based upon work supported, in part, by the National Science Foundation under Grant CNS 1250367; DHS BAA 11-02-TTA 03-0107, and Google. The views and conclusions contained in this document are those of the authors and should not be interpreted as representing the official policies, either expressed or implied, of the Army Research Laboratory, Department of Homeland Security, Google, NSF, Indiana University, Microsoft, or the U.S. Government. The U.S. Government is authorized to reproduce and distribute reprints for Government purposes notwithstanding any copyright notation here on.

Authors' addresses: Z. Dong, Microsoft Corporation, One Microsoft Way, Redmond, WA, 98052; email: Zheng.Dong@microsoft.com; K. Kane, Microsoft Research, One Microsoft Way, Redmond, WA, 98052; email: kkane@microsoft.com; L. J. Camp, School of Informatics and Computing, Indiana University, 901 E 10 th Street, Bloomington, IN, 47408; email: ljcamp@indiana.edu.

Permission to make digital or hard copies of all or part of this work for personal or classroom use is granted without fee provided that copies are not made or distributed for profit or commercial advantage and that copies bear this notice and the full citation on the first page. Copyrights for components of this work owned by others than the author(s) must be honored. Abstracting with credit is permitted. To copy otherwise, or republish, to post on servers or to redistribute to lists, requires prior specific permission and/or a fee. Request permissions from Permissions@acm.org.

2016 Copyright is held by the owner/author(s). Publication rights licensed to ACM.

ACM 2471-2566/2016/09-ART5 $\$ 15.00$

DOI: http://dx.doi.org/10.1145/2975591 
The first assumption is that are no intrinsic restrictions of jurisdiction for the certificate authorities. Any CA can issue public key certificates for any website. While a whitelist or blacklist may exist in some web browsers, the majority of trusted CAs are not subject to any such restrictions.

Second, all CAs are equally trusted. As long as a trust chain can be established without an error to any trusted root, the web browser ignores the difference between a certificate from a large well-known CA such as VeriSign and a certificate by a small regional CA. All CAs are treated equally by default. End users and system administrators are also able to manage trusted roots through the operating system (e.g., Microsoft [2014a]) or in the web browser settings (e.g., Mozilla [2015]).

These assumptions enable websites to switch between trusted CAs without any service interruption. As long as the CAs of the old and new certificates are both trusted, by default there will be no user notification, and the end user is unlikely to notice such a change in CA. In fact, this seamless experience is by design, allowing competition between CAs. (The usability of security indicators and human cognition are beyond the scope of this work.)

These assumptions result in significant security risks. Due to the variations in organizational scale, applicable laws, expertise, and even culture, the operational practices of the CAs can differ significantly. The CAs also face different risks of being compromised. Given these assumptions, one trusted but compromised CA can affect the entire PKI ecosystem. Specifically, with a rogue certificate, the adversary may be able to conduct a TLS man-in-the-middle (MITM) attack between the end user and a fake website. Since the rogue certificate is technically valid and trusted, there will not be automated detection or security warnings generated for such an attack. Unfortunately, given the large number of online websites and the ability to target a specific and limited group of victims, it can be very difficult to detect rogue certificates at their first use, wheras some spear-phishing attacks, such as whaling (which targets high-profile end users), need only one success.

In order to immediately identify a rogue certificate (and potentially the compromise of a CA), we propose a machine-learning approach based on historical certificate observations from the trusted CAs. This approach is based on supervised learning with machine-learning features extracted from the standard X.509 certificate structure. Classification models are built using certificates observed over a matter of weeks and optionally using the connection metadata as well. The resulting models are then used to classify the newly observed certificate as rogue or not.

The advantages of the Deep Neural Networks (DNN) classification model presented here are threefold.

Timeliness. Certificates can be classified immediately using this approach. This allows for faster identification and investigation of potentially rogue certificates, compared with previous compromises.

Correctness. The classification models are able to correctly classify the rogue certificates (see Section 10). We discuss the real-world usefulness of this approach by applying it to the India CCA compromise in July 2014, which happened while this work was underway.

Compliance with current PKI architecture. Deployment of this approach requires no change in the current PKI ecosystem. Training of models is done based on observations available in the infrastructure today and requires no additional cooperation or information from the CAs or browser manufacturers. There is no requirement for additional parties to be added to the architecture, for example, no need for notaries.

The rest of the article is organized as follows. Section 2 reviews the related research in the detection of rogue certificates, application of machine-learning techniques in 
security domains, and other relevant machine-learning topics. Section 3 covers machine-learning background about algorithms that were examined in our work. Section 4 introduces the threat model and describes our corresponding design choices. Sections 5 to 10 describe our system design in detail. Section 5 enumerates the machinelearning features and explains the associated selection criteria. Section 6 describes the datasets for the benign category, which is orders of magnitude larger than the rogue category. Section 7 describes the rogue certificate dataset, the methods typically used to address an unbalanced dataset, and describes our solution to this challenge. Section 8 describes model generation, addressing the choice of DNN as compared to other algorithms. Section 9 reviews a number of metrics that are used for evaluating the performance of these models. Section 10 reports and analyzes the classification results. Section 11 discusses possible deployments, attacker responses, and future directions. Section 12 summarizes and concludes the article.

\section{RELATED WORK}

\subsection{CA Compromises and Rogue Certificate Detection}

The trustworthiness of the certificate authorities is a core tenet of the PKI ecosystem. However, there have been a number of security breaches and mistakes at different CAs. The result has been the issuance of rogue certificates for high-profile websites. For example, in 2011, the CA Comodo was compromised, causing rogue certificates to be issued for large websites such as Microsoft and Google [Hallam-Baker 2011]. In the DigiNotar compromise, over 500 rogue certificates were issued [Fisher 2011]. In 2012, TurkTrust incorrectly coded two end entity certificates as subordinate CA certificates [Microsoft 2013], which gave the holders of those certificates the unintended ability to issue further certificates of their own. Another subordinate CA certificate was wrongly issued by a French government CA in 2013 [ANSSI 2013]. Another recent CA compromise occurred in July 2014 to a subordinate CA of India CCA, causing a number of rogue certificates to be issued for popular domains including Google and Yahoo! [Microsoft 2014b].

There have been several research proposals and implementations to detect rogue certificates. In Perspectives [Wendlandt et al. 2008], end users submit hash values of observed certificates and the associated URLs to a network of servers. This is an implementation of a notary approach, whereby third parties are queried to verify the validity of certificates. A browser plug-in installed by the user then compares this hash value with observations from the same URL across several geographically distributed notary servers. In the system, the end users can choose to submit the hash digests of their observed certificates to any number of user-selected notary servers. The Perspectives browser extension alerts users if a discrepancy is observed. Another centralized certificate notary is maintained by the ICSI from live HTTPS traffic that is passively collected at its participating organizations [Amann et al. 2012]. Based on their dataset, Amann et al. performed a data analysis on the structural differences between benign and rogue certificates observed in previous CA compromises. Their conclusion was that rogue certificates were not consistently distinguishable from benign certificates [Amann et al. 2013]. In comparison, our approach utilizes a richer set of certificate fields and connection metadata as features. We then leverage a DNN classification algorithm to evaluate the underlying relationships between the selected features of rogue and benign certificates, finding systematic differences. Our classification results are reported in Section 8.

Durumeric et al. regularly scan the entire IPv4 address space for certificates and has made several recommendations for the PKI ecosystem [Durumeric et al. 2013]. These proposals largely rely on the presence of a centralized network services. 
The Certificate Pinning technique [Larimer and Root 2012] allows the association of a website with a specific certificate or set of public keys. This information can be included as part of the browser or obtained via a notary query when a website is first visited. This approach, however, is not scalable. Pinning on the first visit is vulnerable to adversaries who are capable of providing a fraudulent certificate to an end user on the first visit. Like other whitelist approaches, completeness is a challenge particularly given the long tail distribution of websites.

Likewise, Certificate Transparency [Laurie et al. 2013] (CT) provides publiclyqueryable logs of issued certificates. It relies upon the participation of end users via browser plug-ins, website owners voluntarily submitting certificates they are using, and participating Certificate Authorities submitting certificates upon issuance. Certificate Transparency leverages the Merkle Hash Tree [Merkle 1988] to allow efficient proofs of the integrity of the logs. CT itself is a data source that guarantees any certificates submitted to it will remain visible and unaltered, but leaves detection of mis-issued certificates to Monitors and trusted third parties. For example, much as we do as described in Section 5, a Monitor could extract properties of a certificate and analyze it for anomalies, or a website owner could regularly query the log for owned domain names and compare the list of certificates to a list they have of certificates they have legitimately purchased. The precise mechanisms of detection are beyond CT's scope, but like our approach, depend upon a sufficiently high level of visibility into the PKI ecosystem. The effectiveness of CT also relies significantly on the rate of participation of the Certificate Authorities providing faithful logs of their issued certificates in addition to user-provided telemetry; we rely only on the user-provided telemetry. We discuss CT as a data source in Section 11.

DANE (DNS-based Authentication of Named Entities [L. Barnes 2011]) allows certificate information to be included in the domain record in a DNS server, protected by DNSSEC. In addition to relying on trusted CAs, this technique depends on the trustworthiness of the domain registrar, the owner of the DNS servers, and the widespread adoption of DNSSEC.

In addition to public key certificates themselves, vulnerabilities can exist in various implementations of SSL and TLS. To identify these vulnerabilities Brubaker et al. [2014] designed the FrankenCerts mechanism so a large number of artificial rogue certificates can be generated for adversarial testing. Working with Facebook to detect MITM attacks, Huang et al. [2014] performed a large scale analysis of the public key certificates the end users encountered when connecting with Facebook. Certificates were collected from a group of sampled Facebook users through a Flash player plug-in to the socket policy server. Over six thousand uses of suspect certificates were discovered during their experiment as part of 3 million connections to Facebook. However, they did not mention the number of unique certificates that were observed, nor was the number of connections made behind a corporate firewall discussed. This approach is also not scalable to every online website, and as the authors note, either a firewall already in place or an adversary may foil the telemetry employed by their technique.

\subsection{Machine Learning in Security}

Machine-learning techniques have been applied in various security domains. There are two general types of machine-learning approaches: supervised learning and unsupervised learning. Supervised learning is often used to perform classification or regression tasks for new instances. It relies on a training dataset of instances labeled with the correct category. The learning algorithm then tries to model the underlying relationships between a number of predefined features and the categories. If the training dataset is not available, that unsupervised learning algorithms can still divide the dataset into a number of clusters, based on the distances between instances. 
Compared to the signature-based blacklists and whitelists, machine-learning-based approaches can often achieve a higher detection rate for quickly evolving cyberattacks such as malware and botnets. With a carefully selected feature set, machinelearning models can capture the underlying logic or connections that traditional approaches would otherwise overlook. For instance, Bailey et al. analyzed and extracted machine-learning features from the operations performed by malware and demonstrated the use of clustering analysis to detect polymorphic malware [Bailey et al. 2007]. Gu et al. performed clustering analysis on monitored network traffic to identify potential botnets [Gu et al. 2008]. Features were selected from the connections between the two communication parties and properties of the outbound network traffic.

Machine learning has also been used to analyze access control. Association data mining has been used to identify access control deviations, with the assumption that these access control anomalies were errors and not insider attacks [Bauer et al. 2011]. Similar to our results, one advantage of the technique was reduction in the lag time for identification of anomalies [Mazurek et al. 2013].

To address assignments in role-based access control Streich et al. used Multi-Agent Clustering to allocate access rights more accurately [Streich et al. 2009]. The authors found that this was particularly applicable to timely assignment of correct access to new employees. The original dataset was augmented with artificial data in order to improve accuracy using Bayesian techniques. In the case of rogue and benign certificates no certificate can be assigned to more than one cluster, so Multi-Agent Clustering was not evaluated in our work. Similar to our work, artificial instances were generated using standard machine-learning techniques.

PGBGP is a mechanism that is similar to our proposed machine-learning approach, although it is applied to the identification of most trustworthy Boarder Gateway Protocol (BGP) routes [Karlin et al. 2006]. PGBGP considers a combination of several factors when ranking the BGP routes but utilizes only simple rule matching techniques when making decisions.

Phishing detection is another security domain where machine learning has been applied. Previous research examined both phishing emails (source) and phishing websites (destination). For example, Basnet et al. tested features extracted from the format and contents of emails and were able to detect the majority of the 500 phishing email samples [Basnet et al. 2008]. Several other proposals extracted features from the page content. For example, Rosiello et al. constructed the HTML Document Object Model (DOM) trees for phishing and benign webpages, and used measurements such as layout similarity for the detection of phishing pages [Rosiello et al. 2007]. CANTINA+ [Xiang et al. 2011] is a multi-layer approach to extract features automatically from the DOM of a page. Additional indicators of phishing may be obtained from the page URLs. Garera et al. created a detection technique based on different obfuscation observations and misspellings of the URL [Garera et al. 2007]. Ma et al. combined the textual features of the URL (e.g. special characters, word appearance) and host information [Ma et al. 2009]. Using Google's blacklist, Whittaker et al. built phishing detection systems using features extracted from the page, such as the URL and page content [Whittaker et al. 2010]. Mishari et al. examined public key certificates using machine learning to distinguish phishing websites with typo-squatting URLs using TLS [Mishari et al. 2009]. Classifying rogue certificates is more challenging because the available training data for rogue examples is far smaller than the available training data for phishing sites. One of our contributions is an approach to address this data imbalance problem. The performance of classification algorithms may be negatively affected if the number of instances of one category is significantly smaller than the other [Chawla 2005]. We further discuss this issue in Section 7.1. Conversely, classifying rogue certificates may be less challenging because the problem is much more structured. This structure offers 
the potential for more accurate results. In addition, the results can be more effective in operation because the attackers are more restricted in the ability to alter certificates in comparison with the malleable structure of phishing attacks.

Machine learning is widely deployed to identify spam emails. Recent work has expanded this to SMS. A client-side machine-learning approach with a binary classifier was used to detect SMS spam in Duman et al. [2014]. The results were promising, with an increase in accuracy of spam identification from $50 \%$ in current applications to up to $98 \%$ in the reported two-level detection method with a Support Vector Machine (SVM) [Cortes and Vapnik 1995].

Machine learning has been used in attacks as well as defense. Combining a Bayesian classifier and SVM, Dyer et al. identified destination websites over encrypted traffic with $98 \%$ accuracy [Dyer et al. 2012].

One common criticism of machine-learning-based security mechanisms is that the adversary can often adapt their attacks to subvert the new countermeasures. An early discussion on the attacks against machine-learning-based security mechanisms was provided by Huang et al. [2011]. Wang et al. studied adjusted behaviors (and therefore machine-learning features) of malicious crowdsourcing workers in a large online social network after the deployment of a machine-learning-based detection system. Their research found that in this particular scenario the machine-learning algorithm and the knowledge of the attacker about the model could all contribute to the robustness of the machine-learning approach [Wang et al. 2014]. Previous work detailing challenges in using machine learning for security domains [Sommer and Paxson 2010] (albeit in intrusion detection systems) noted the need for defining the context of application. Our application of machine learning to rogue certificates meets the identified criteria for machine-learning domains. The strict requirements for certificate structure and the specific domain chosen argue for machine learning as an effective approach. The variability in data is far smaller in the certificate case compared to the IDS domain. The creation of artificial instances addresses the lack of rogue data. In part because of these differences, we were able to demonstrate operational success through the timely identification of the India CCA rogue certificates.

It would be difficult for an adversary to adjust and circumvent our rogue certificate detection mechanism for five reasons. First of all, public key certificates have to comply with the X.509 standard. Every certificate field needs to have a legitimate and valid value to pass the validation check of the web browsers or non-browser applications. The adversary may not be able to put arbitrary values in all fields even if they can perform operations with the CA's private key.

Second, there are certain fields that an adversary needs to change for the rogue certificates to be potentially useful. For example, to prevent a rogue certificate from being discovered, an adversary may intentionally remove or modify the revocation information in a certificate. That itself can be a strong indicator of a rogue certificate if the compromised $\mathrm{CA}$ is otherwise consistent in implementing revocation.

Third, depending on the specific attack, the adversary may only be able to perform some certain operations on behalf of the compromised CA. In this case, certificate extensions such as Basic Constraints, Key Usage or Extended Key Usage may be different than previous observations.

Fourth, in previous attacks the adversary has preserved the current date in the Not Valid Before field of the certificate, as seen in incidents such as Diginotar [Fisher 2011], TurkTrust [Microsoft 2013] and so on. The Not Valid Before is used in one feature, which is then combined with all other features to generate the classification result. The feature that is used in our mechanism calculates the difference between the Not Valid Before date and our first observation date. Thus a pre-dated Not Valid Before date would also be suspicious because of the relationship between our infrastructures date and point of observation and the Not Valid Before date. While an attacker can change 
the Not Valid Before date, the attacker cannot control our first point of observation. The majority of previously discovered fraudulent certificates were newly issued, with only a few days between the issuance and the observation dates. This feature is not the only determining factor of the classification.

Fifth, our machine-learning features can be improved over time in the course of normal operation of possible deployments, which we discuss in Section 11.1. For example, an organization can change the importance of feature or add their own features to identify targeted attacks. This is because our approach is repeatable and thus can be optimized to different organizations.

We also discuss potential attacker responses in Section 11.2.

\section{MACHINE LEARNING BACKGROUND: DEEP NEURAL NETWORK AND OTHER TESTED ALGORITHMS}

From a machine-learning perspective, the classification of benign and rogue certificates is a supervised-learning problem, specifically with a predefined set of machine-learning features and a training dataset with the known category label (rogue or not) for each instance in the dataset. A machine-learning model is generated using the algorithm and training dataset. This model is then used for predicting the category of testing instances without the category labels.

We compared the performance of machine-learning models with several supervisedlearning algorithms. The DNN [Bengio 2009] was the top algorithm to model complex relationships between the low-level inputs and the predicted category. DNN provided higher accuracy with a shorter training period. DNN has been widely utilized to solve complex problems such as natural language processing, as well as voice and face recognition. In our approach, we utilize DNN to identify higher-level connections between machine-learning features that are directly derived from the certificate fields and the category of certificates.

Like traditional neural networks, the DNN model contains one or more intermediate hidden layers, between the input and output nodes (neurons) of the model. Nodes on different layers are connected from the lower layers (input) to the higher layers (output), such that each node connects to one or more neighboring nodes in the adjacent layers. Through each node, an activation function maps the inputs (weighted values of the connected lower-level nodes and a constant bias) to the output of the node, which then becomes a component of a higher-level node's input.

Traditional neural networks use backpropagation and gradient descent methods to find an optimal combination of weights and biases for the activation functions of the entire neural network. The classification performance of traditional neural networks can become less optimal with the addition of more layers between the input and output.

DNN enables more complex neural network to be built with higher classification performance, particularly with more than one hidden layer. In addition to backpropagation and the stochastic gradient descent (SGD) [Bottou 1991] methods, training deep neural networks also differs from that of traditional simple neural networks in terms of procedures. The training of DNN consists of two stages: pre-training and fine-tuning. In the pre-training stage, the algorithm starts with the first hidden layer. The goal of each step in this stage is to determine initial weights and bias for nodes on the lower layer. Each step in the pre-training stage is an unsupervised learning and focuses only on one layer at a time. At the end of the step, the algorithm moves one layer up until reaching the highest hidden layer. In the second stage the algorithm makes further adjustments to the initial weights on each layer with backpropagation. The unsupervised pre-training stage can greatly improve the overall performance of the trained neural network [Erhan et al. 2010], enabling effective training for deeper (more than one hidden layer) networks. 

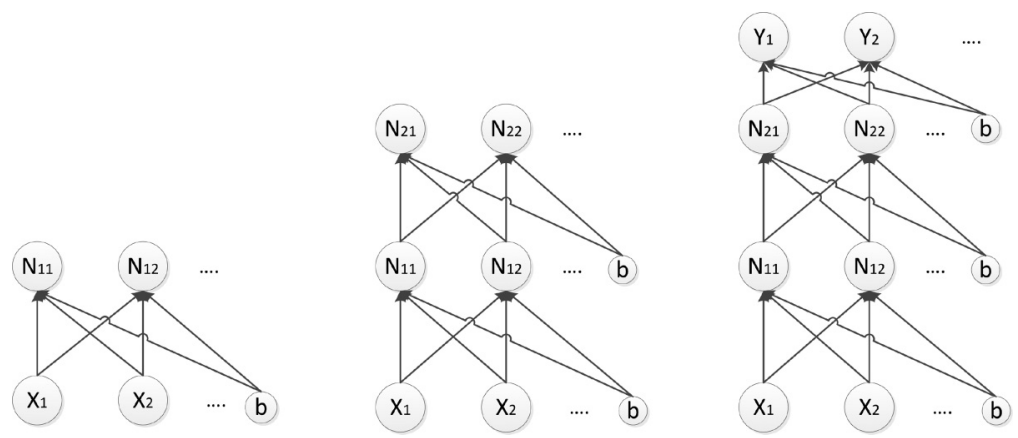

Fig. 1. Training procedure of a DNN model.

Figure 1 demonstrates the procedure of training a DNN model with two hidden layers. The first two subfigures are in the unsupervised pre-training stage to determine the initial weights, in which one layer is focused in each round. Once completed, it adds back the output layer and performs a supervised learning, as shown in the third subfigure. The methods of evaluating the results of a machine-learning approach are precision, recall, and accuracy as well as the Area Under the Receiver Operating Characteristic (ROC) Curve (AUC). All of the standard measures are defined in Section 9 and reported in Section 8.

In general, DNN is capable of solving more difficult problems than traditional neural networks, with more complex models and within a reasonable training period. We chose $\mathrm{DNN}$ as the classification algorithm for this application due to its superior performance in our experiments.

In addition to DNN, we have also tested the performance of Random Forest, Logistic Regression and SVM algorithms. Random Forest [Breiman 2001] is an ensemblelearning algorithm that builds a number of decision trees and determines the category of an instance through a majority vote of those trees. To construct a decision tree in the forest, a fixed number ( $\mathrm{n}$ ) of instances are randomly selected (with replacement) from the larger training dataset for model training. To decide on how to split the decision tree, another fixed number $(\mathrm{m})$ of features are randomly selected each round from the entire feature set. Random Forest was also among the top performed algorithms in our experiment, but it required twice the training time of DNN.

Logistic Regression [Le Cessie and Van Houwelingen 1992] is a regression analysis algorithm that tries to represent the underlying relationships between the feature set and the category using one logistic function for each category. In our experiment, logistic regression required a training period similar to DNN but produced lower accuracy for both categories (rogue and benign).

The SVM [Cortes and Vapnik 1995] approach tries to find one or more hyper-planes to separate the instances into different categories in a high-dimensional space. According to our experiment the model-building process took longer than logistic regression and yielded a comparable classification performance. While SVM has been found to perform well in hierarchical domains with clear structural taxonomies [Liu et al. 2005], here the DNN approach was found to have superior accuracy.

\section{SYSTEM DESIGN}

\subsection{Threat Model}

We make the assumption of a strong adversary who is capable of controlling the certificate issuance of a CA or performing a signature operation with a private key of the CA. The result in either case is a certificate that will be trusted by any browser 


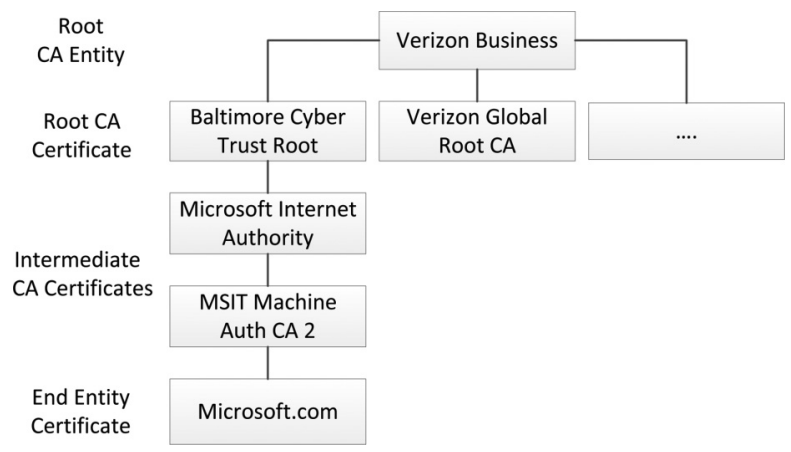

Fig. 2. Example of a root CA entity and its hierarchy-Microsoft.com.

or other application that trusts the CA but that the CA would not have issued under normal business processes. We specifically exclude certificates that fail client-side validation for any reason as they are not representative of the rogue certificates we wish to identify. Thus they are not instructive in training a model. The resulting assumptions about certificates are that these comply with the X.509 format, are within their stated validity periods, have a valid cryptographic signature, and that signature is from a CA through which a chain of trust exists to a trusted root.

Having a rogue certificate enables a range of attacks. One possible attack is the MITM attack. Unlike typo-squatting attacks, which depend on the user to err in a predictable manner, a rogue certificate enables an attack using a legitimate domain name. In this case, the user seeks a legitimate website (such as Google, Yahoo!, or Microsoft) and commits no navigation error. Instead, that user is misdirected to another site. There is no need for typo squatting in this case. The rogue certificate matches the domain name, has appropriate credentials, and the MITM attacker can observe all interactions between the client and the site. Of course, MITM attacks without rogue certificates are also possible. However, in this case either the certificate does not match the domain, the website is not SSL/TLS enabled, or the certificate does not chain up to a trusted root.

A rogue certificate can be used to convince victims to download updates or software that appear to come from a trusted third-party. This was most famously implemented in Stuxnet [Falliere et al. 2011]. However, in the case of Stuxnet, there was not a rogue certificate for the domain itself; rather, the certificate was for the malicious code.

\subsection{Design Goal}

Our approach identifies technically valid certificates that are potentially malicious. The approach relies solely on the certificate of a website and, if available, the associated page content or URL.

Our machine-learning models are focused around the certificate authorities. However, given the large number of intermediate CAs and possible variations of their issuing behaviors, it can be difficult to create one classification model (with reasonable size) that covers all CAs. At the same time, it is also impractical to train one classification model for every intermediate CA. Training for every intermediate CA would require infeasible commitments to model training, maintenance, and predictions.

After evaluating several possible options, we chose a balanced approach. We train one classification model for every Root CA entity. "Root CA Entity" refers to the corporation or organization that operates one or more root CAs. Figure 2 is one example of a root CA entity and its hierarchy. In this particular case, the classification model covers all root CAs operated by Verizon Business, the intermediate CAs, and the end-entity 


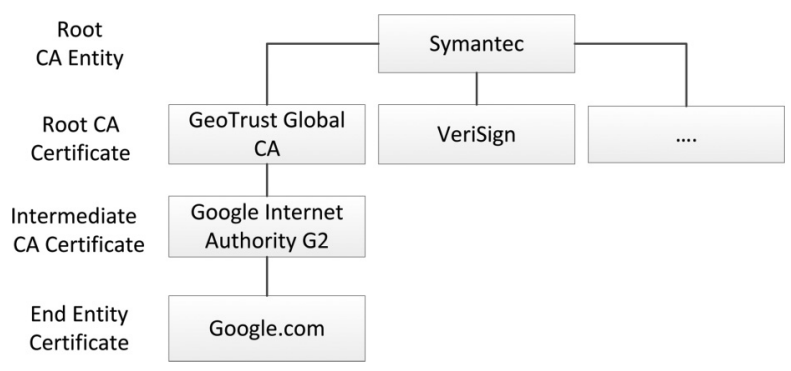

Fig. 3. Second example of a root CA entity and its hierarchy-Google.com.

certificates. As issuance processes do change over time, the machine-learning models are updated regularly at a certain time interval. Based on our experiments, we found a weekly update to be sufficient. (We further discuss deployment in Section 8.)

Figure 3 shows the hierarchy of CAs of Google.com. Note that in this case, our models are not built for GeoTrust, which is the root CA. Instead, a machine-learning model is trained for the CA entity Symantec, the company that operates the root CA. This model will also cover other certificates issued under root CAs operated by Symantec, such as VeriSign.

We start by defining a set of machine-learning features. These features are extracted from the standard and optional certificate fields, and the certificate trust chains. If available, then metadata about the connection, such as from where the certificate was seen and URL, are included. This process occurs only once at the beginning of the system deployment. A complete description of the machine-learning features is listed in Section 5. Note that with the approach described here, any organization with machine-learning expertise and a web crawler (to obtain a dataset) can train their own machine-learning models with the historically observed certificates. Organizations that host their own CAs can use this approach to determine if they themselves have been targeted by a rogue certificate. In case of a CA compromise, our approach is capable of providing more complete coverage than the internal audit logs.

Our proposed approach simultaneously achieves correctness, timeliness, and completeness.

Correctness is a fundamental requirement for any system that detects anomalous behaviors. Correctness can be directly evaluated by the accuracy, precision, and recall rates of the classification.

Traditional centralized approaches often do not perform well in timeliness, as the difficulty of identifying rogue certificates results in a lag between the first use of a rogue certificate and the discovery of the certificate. There is additional delay until the rogue certificate is blacklisted on the end user's machine.

Completeness is another challenge faced by traditional signature-based security solutions. TLS MITM attacks are often closely related to spear-phishing attacks and target only at a small group of users. It is therefore difficult to discover and blacklist the targeted rogue certificates. However, our approach generates machine-learning models for every root CA entity that is trusted by the major operating systems and web browsers. Therefore this approach is able to cover all certificates following the chain of trust, even certificates that are not issued at the time of model generation.

\subsection{System Components}

We began by designing an experimental system applicable to any root CA entity. The system consists of the following components: machine-learning feature extractor, rogue certificates simulator, data splitter, model generator, and performance evaluator. The 


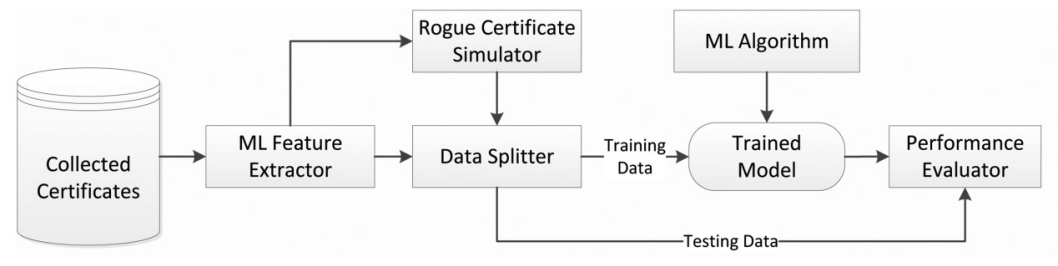

Fig. 4. Experimental system design.

interaction of system components is shown in Figure 4, and we introduce the design of each component as follows.

Machine-learning feature extractor. According to the predefined feature set, this component extracts values of machine-learning features from the collected public key certificates.

Rogue certificate simulator. We began with a small sample of rogue certificates. We then created an innovative approach to generate artificial rogue instances based on instances in the benign category. This is required by the scarcity of observed rogue certificates and their insufficient coverage of potential attacks. We discuss this process in detail in Section 7.

Data splitter. This component separates the training and testing datasets for both categories: benign and rogue. For the experimental setting, we randomly selected (without replacement) $70 \%$ of the instances for training and used the remaining $30 \%$ for testing in both categories (benign and rogue).

Model generator. A classification model is generated by applying a machinelearning algorithm to the training datasets in the benign and rogue categories.

Performance evaluator. The testing datasets of both categories are provided to the generated model. Performance metrics including accuracy, precision, and recall are calculated by this component.

\section{FEATURE SELECTION}

In this section, we list the machine-learning features utilized in the classifications and the reasons that they were selected. We group these features into nine categories: certificate properties, issuer, subject, validity, revocation, extensions, blacklist, certificate chain, and connection information. These features were selected as a set that should be representative of a CA's issuance practices and should be consistent across certificates from a particular root CA entity.

\section{A. Certificate Properties}

-Version. The version of the certificate indicates the standard structure of the certificate. While it is recommended that all certificates be at least X.509 Version 3, we have seen a considerable number of certificates that are still at X.509 Version 1 in our data collection (until August 2014), with no certificate extensions (as these are not supported by version 1). Our observed V1 certificates were either older root CA certificates or untrusted and usually self-signed end-entity certificates not issued by a trusted CA. According to the Baseline Requirements of the CA/Browser Forum [Forum 2015], it is no longer an acceptable practice to issue V1 certificates because of their limitations; any new ones seen from a trusted CA would therefore be immediately suspicious.

-The length of the certificate serial number. A serial number is assigned by the issuer to every certificate as a unique identifier. While there is no guarantee that the serial numbers are unique across CAs, the length of the serial numbers from a specific CA is fairly consistent. 
-Signature algorithm. This extension includes both the algorithm to create message digest and the algorithm to generate digital signature of the message digest. As observed in our dataset, SHA1 and RSA are the most utilized algorithms.

-Basic Constraints. This certificate extension includes two components. The first is the CA flag, stating whether this is a CA certificate or an end-entity certificate. The second is the maximum path length, which in the case of CA certificates restricts the number of additional intermediate CAs that can follow this CA in a chain. The path length has no meaning for end-entity certificates. A maximum path length of zero means that no intermediates may follow the certificate, indicating that the particular CA can only issue end-entity certificates. If the path length is absent but the CA flag is true, then there is no limit. Deviations in the construction of this extension have led to some previous attacks. Several CA compromises that we listed in Section 2.1 involve incorrect encoding of the CA flag, such as the TurkTrust compromise and the French Government CA incident. Should a mis-issued subordinate certificate be exploited by an adversary to issue further end-entity certificates, the length of the trusted chain can become an indicator since it may be one or more level(s) longer than the certificates issued during normal legitimate business process.

- Key Usage. This is a certificate extension that defines the intended purpose of the public key embedded in the certificate. According to the IETF RFC 5280 [IETF 2008] there are nine legitimate values that can be used in this extension: digital signature, non-repudiation, key encipherment, data encipherment, key agreement, key cert sign, CRL sign, encipher only, and decipher only.

-Extended Key Usage (EKU). This extension provides a richer set of potential key usages, such as server authentication, client authentication, and code signing. The EKU can work together with the Key Usage extension to enforce a comprehensive restriction on how a public key may be used. For TLS and code signing, two common uses of certificates, the expected sets of values in both Key Usage and Extended Key Usage are small.

\section{B. Certificate Issuer}

-Distinguished Name (DN). DN is a standard approach to assert the identity of an entity. In an X.509 certificate, both the issuer and the subject's identity information are listed as distinguished names. There are several (optional) components of the $\mathrm{DN}$ that are commonly present in the X.509 certificate: common name (CN), organization name $(\mathrm{O})$, organizational unit name (OU), email (E), locality $(\mathrm{L})$, state $(\mathrm{S})$, and country (C). Each of these components is used as a machine-learning feature. The issuer DNs stay constant over a long period of time. Given that the actual values of the issuer DNs are both indicative and constant, they are useful machine-learning features.

-Authority Key Identifier. It is possible that multiple public/private key pairs are used by a CA in normal operations. This extension indicates the specific public key that corresponds to the private key of the CA for the generation of the digital signature. It enables quick reconstruction of a trusted path and can also become an important indicator of the issuing patterns.

-Issuer Alternative Name (IAN). In addition to the identity information listed in the standard DNs, the CA may choose to have additional information, such as the DNS address, or Universal Resource Identifier (URI), included in this certificate extension. In some cases we used the actual content of the IAN; in other cases we used only the existence of the IAN extension. 


\section{Certificate Subject}

-Distinguished Name. The structure of the subject's DNs is similar to those of the certificate issuer. Issuer and subject DNs, however, are treated differently as machine-learning features. Every trusted CA may issue certificates to any subject and the subject may also switch between CAs. Due to the huge set of possible combination of subject sub-fields, only the country of the subject is utilized as a machinelearning feature. For all other sub-fields, we only use the existence of each of the other sub-field as a feature and not their value.

-Subject Key Identifier (SKI). Like the Authority Key Identifier extension, it is possible for a single subject to be issued multiple public key certificates. The identifier of the subject's public key provides a fast way of building a trusted chain. Our approach includes the existence of the $S K I$ as a machine-learning feature but not the content.

—Subject Alternative Name. Similarly to the Issuer Alternative Name extension, the issuer may choose to provide detailed information about the subject in this extension in addition to the DNs. One useful scenario of this extension would be to list all domain names (often owned by the same organization) to which the certificate is issued. Our approach records the number of entries of the subject's alternative name as a machine-learning feature but not the names themselves.

\section{Validity}

Two required fields exist in the X.509 certificate to define its time validity: Not Valid Before and Not Valid After. To ensure that our approach is applicable for all collected certificates, we calculate three machine-learning features from the two fields and the date of observation. First, we consider the entire length of the validity period. Second, we include the number of days between the observation and Not Valid Before as a feature. Third, we evaluate based on number of days between the observation and the Not Valid After. These relative measures are frequently consistent in our observations making them suitable machine-learning features, whereas the actual dates nearly all differ. In previous compromises, when a rogue certificate has been discovered, frequently its Not Valid Before date was recent.

\section{E. Revocation}

Currently, two revocation approaches are commonly deployed by the CAs: Certificate Revocation List (CRL) and Online Certificate Status Protocol (OCSP). The CA may utilize one approach, or both, or neither. CRL relies on the CRL file address listed in the CRL Distribution Point extension. The URL of the OCSP server is indicated in the Authority Information Access extension. These two revocation methods are both valid approaches to revoke a rogue certificate when it is discovered. An adversary may have an incentive to modify or remove the authentic revocation information to avoid quick blacklisting. Through an analysis of previous discovered rogue certificates, the change of revocation information can create a strong indicator of a rogue certificate. Both the existence of the CRL or OCSP information and the values of these two extensions are currently used by our approach as machine-learning features. Note that they are not the only features that determine the classification result. Instead, the classification is conducted based on the combination of all machine-learning features.

\section{F. Blacklist}

Although it is rare, certificates may be issued to internal entities by trusted authorities. However, it may become a security threat when the subject's identity is not clearly defined. We created a list of entities that should not be signed. This list includes localonly host names such as Localhost, as well as private IP addresses such as 192.168.x.x. 


\section{G. Certificate Chain}

The end-entity certificate is often the one that attracts attention. However, the entire chain of trust can sometimes provide additional clues on the maliciousness of a certificate. Our approach records the length of the certificate chain as one important feature. Through a comparison of historical observations, this feature would be able to label attacks when a subordinate CA certificate is mistakenly issued by the CA, possibly connecting with additional issuance of rogue certificates.

Extended Validation (EV) is another important indicator. Recipients of EV certificates tend to be more vetted than normal recipients of standard certificates. CAs have different standards for additional verifications on the subjects' identities, yet every CA has more validation for EVs than standard certificates. Thus we include the identification of $E V$ certificates as a feature.

\section{H. Connection Metadata}

Through collaboration with a major browser manufacturer, we have access to the following anonymized connection metadata from the TLS connections provided by telemetry from users of that browser. Including the connection-related features increased the classification performance, with the additional origin and destination information. However, our classification mechanism still achieved high performance when such information is unavailable, as examined by several detection experiments, including the India CCA compromise incident, which we discuss in Section 10. Features in this group are listed below.

-Accept Language. This feature is the displayed language of the web page on the web browser.

-Page URL Level and Country. These features include the number of levels of the page URL and the geo-located country of the corresponding IP address.

- Referrer URL Level and Country. These features include the number of levels of the referring page URL and the geo-located country of the corresponding IP address.

-DNS Country. This feature indicates the location of the DNS server where the initiator makes this request.

\section{Other Extensions}

In addition to the extensions mentioned above, our approach evaluates the following features of certificates as machine-learning features.

- The total number of extensions as well as the number of extensions that are not covered in other features. These two features capture the common certificate extensions that a CA would include in a certificate and the number of extensions that are not commonly seen, respectively.

-Whether each extension is labeled as "critical." There is no strict requirement as to critical extensions but recommendations are made by the IETF that certain certificate extensions (if they exist) should be labeled as critical. If a certificate deviates from the previously observed issuance behavior of a particular CA on this, then it is more likely to be a rogue certificate. This feature demonstrates the CA pattern of labeling extensions.

\section{DATA COLLECTION}

In this section, we introduce the data sources and approaches that were utilized in the performance evaluation of our machine-learning-based rogue certificate detection mechanism.

We collaborated with a major browser manufacturer and obtained access to several datasets. The first dataset is the daily web crawl of public key certificate chains from 
Table I. Certificates Issued by Top 10 Root CA Entities

\begin{tabular}{ccc}
\hline Root CA Entity & Top 1M Site Certs & User Submitted Certs \\
\hline Symantec & 253681 & 368035 \\
Comodo & 133006 & 150594 \\
GoDaddy & 108456 & 171324 \\
GlobalSign & 89691 & 65675 \\
DigiCert & 18990 & 55276 \\
StartCom & 15931 & 12060 \\
Verizon & 9230 & 26796 \\
Entrust & 6876 & 29198 \\
Trustwave & 4590 & 5884 \\
SECOM & 4261 & 8140 \\
\# Certs Total & 889849 & 2516155 \\
\hline \# Certs from Top & 644712 & 892982 \\
Ten Root CA Entities & $72 \%$ & $35 \%$ \\
\hline$\%$ of Certs from Top & & \\
Ten Root CA Entities & & \\
\hline
\end{tabular}

the top $100 \mathrm{~K}$ websites, according to Alexa. ${ }^{1}$ Certificates from the top 1 million websites were also downloaded on a weekly basis. A third dataset includes the certificates and connection metadata seen by users of the web browser. These users voluntarily participated in a program to protect their online browsing and agreed to share their anonymized observations, including certificate data for HTTPS connections. The browsing information is sampled at the user's end at an extremely low rate. But because of the adoption rate from the large user base, this provides sufficient data for the analysis without exposing much about a single user's browsing. The following connection information is recorded for every session: the Accept-Language header, page URL, country where the IP address of the website resides in, the referrer URL and country, the DNS information, and so on. Certificate chains are recorded if the session utilizes HTTPS. Machine-learning features were extracted according to this metadata in addition to the certificate fields.

Certificates from the top 1 million sites were collected ad hoc until April 19, 2014, when collections happened automatically on a weekly cadence. As of November 1, 2014 , we observed 889,849 unique certificate instances in this dataset. The size of the dataset has been increasing steadily at a relatively constant rate, consistent with sites renewing certificates and changing CAs. On average, our data collection observed 7,710 new unique certificates per week.

Certificates submitted by end users are done so with a low sampling rate and connection metadata is scrubbed of personally identifying information before being added to this dataset to protect an individual user's privacy. As of November 4, 2014, the number of accumulated distinct certificates reached $2,516,155$. Since the submitted certificates are not necessarily from the top 1 million sites, the weekly growth rate of this dataset was much faster than the former dataset, at 60,103 per week. These certificates do include untrusted certificates that we do not consider rogue, because they do not chain up to a trusted root CA and so are excluded from our training and testing sets.

Among the total collected certificates, $72.5 \%$ of the top 1 million certificates were issued by a top 10 root CA entity, while $35.5 \%$ of the end user submitted certificates were from the top 10 root CA entities, as shown in Table I. According to the table, the numbers of issued certificates differ significantly even for the 10 root CA entities that issued the most certificates. Considering the underlying patterns for the potentially

\footnotetext{
$\overline{{ }^{1} \mathrm{http}: / / \mathrm{s} 3 . a m a z o n a w s . c o m / a l e x a-s t a t i c / t o p-1 m . c s v . z i p . ~}$
} 
large number of root CAs and intermediate CAs under the root CA entities, it is crucial to generate one model for each root CA entity. As a proof-of-concept, we only report the results of the top 10 root CA entities in this article as they have represented a significant portion of the observed certificates. Generating machine-learning models for other smaller CAs can follow the same procedure. As we described in Section 4.2, each root CA entity has a corresponding trained model, and the model is used solely for the purpose of identifying fraudulent certificates that claim to be issued by one of the CAs of the root CA entity.

Collected and submitted certificates were partitioned into training and testing examples of the benign category in the classification. To validate the benign certificates, we first examined the validity fields to detect certificates that were not yet valid or expired and the trust status (i.e., self-signed certificates or certificates from untrusted roots). We further narrowed the trusted certificates down to those that also appeared in the Top 1 million dataset. Removing these long-tail (and less likely to be targets) sites improved the performance of the classifier. As the last step in cleaning the benign dataset, only the trusted certificates from each CA were considered for each instantiating of the model. Unless otherwise noted, model results are presented in the aggregate as well as for individual CAs. (Note that in the India CCA case the identification was partially based on the fact that this CA had never before issued certificates to sites outside of India, as we discuss in Section 10.)

\section{ROGUE CERTIFICATE SIMULATOR}

Compared to the number of benign certificates issued, the number of rogue certificates is extremely small. Very few of those complete rogue certificate strings have been disclosed to the public. For example, although approximately 500 rogue certificates were issued during the compromise of DigiNotar, a significant portion of the certificates (for example, certificate extensions) has not been included in the public report [Tor 2011]. There are orders of magnitude more benign than rogue certificates.

In our proposed machine-learning-based solution, we try to classify the certificate instances into two categories: rogue and benign. Traditional binary classification algorithms would achieve their optimal performance when the datasets of each category are roughly equal in size. Most algorithms try to maximize the overall accuracy of the classification and therefore tend to classify an unknown instance as the larger category. This reflects an implicit assumption that the costs of misclassifications in both categories are the same. Such an assumption does not apply here.

\subsection{Imbalanced Datasets}

There are standard approaches to address the problem of imbalanced datasets: over-sampling and under-sampling. Over-sampling requires replicating instances in the smaller class, through random selection or generating synthetic instances from the existing minority class [Chawla et al. 2002]. In contrast, under-sampling alleviates the imbalance by sampling instances from the larger class only and uses these instances for classification. As over-sampling and under-sampling are applicable to different classes, they can be utilized simultaneously to construct two new classes of equal size. This combined solution improves the classification performance [Schistad Solberg and Solberg 1996].

However, over-sampling and under-sampling approaches suffer from several drawbacks. When under-sampling from the majority class (benign certificate instances), it is always a challenge to find the most representative instances in the class. With only a small portion of instances being included, it is possible that certain important patterns are not included in the sample. Likewise, over-sampling on smaller category can limit in the coverage of possible rogue certificates. For example, rogue 
certificates identified during a known CA compromise will not be a reliable source of training the classification models, since new rogue certificates might not follow the same pattern.

As it is impossible to obtain sufficient amount of training data for the rogue category, we then considered generating artificial rogue instances for the classification. The use of models or artificial data to understand the world is an important aspect of simplifying complex and potentially unintuitive data [Toon 2012; Weisberg 2013]. In many cases, there are aspects of data that are related to the subject of interest but are not direct measurements. However, constructing a model that ties these components together in a meaningful way can lead to reasonable approximations of the specific measure of interest. This has been used effectively in epidemiology, where often the number of parameters of interest make direct analysis difficult and the data is increasingly noisy [Fumanelli et al. 2012; Kelley and Camp 2012]. In these cases, a reasonable model is created and the synthetic data from the model are used to reason about the quantity in question. As more or better data become available later, the model can be examined to see what it captures and where it fails [Weisberg 2013].

In terms of generating artificial certificates, FrankenCerts [Brubaker et al. 2014] provided an effective approach to generate rogue certificates for adversarial testing. The primary idea of FrankenCerts is to select values of certificate fields from previously observed certificates and extensions from completely random values. The artificial instances in our training set needed to meet four criteria: small distances from benign instances, legitimate feature values, generated with processing requirements suitable for deployment, and consistent with patterns of the corresponding CA. We will further discuss them in the next few paragraphs.

In this work, the number of previously observed rogue certificates is significantly smaller than the daily collected benign certificates. It is therefore obvious that the rogue certificates are the minority class in the binary classification. Note that the generation of artificial instances for the minority class (usually attacks or fraudulent entries) already exist in machine-learning-based security to address the problem of imbalanced training set. Our designed method was influenced by three previous approaches: Distributed, Resampling, and Negative Selection. All three approaches can be used for generating artificial instances for the minor class through mutations from the majority class instances.

Artificial attack instances were generated in the Distributed approach [Fan et al. 2004 ] to identify unknown network intrusions. The approach mutates one feature at a time. For each instance with a less common feature value, the algorithm replaces the value with a randomly generated one and replicates the other features. The newly generated instance is then considered as an artificial rogue instance. The process is repeated until all benign instances have been covered. This algorithm runs fast, with artificial rogue instances generated that are extremely similar to the benign certificates. However, since each artificial rogue instance is only one feature away from a benign instance, it is highly possible that the generated rogue instance also exists in the benign set.

The second approach we considered was Resampling [Theiler and Cai 2003]. It was designed as a general approach to identify the unknown anomalous instances. The primary idea of this approach is to combine feature values from randomly selected benign instances. Specifically, the approach randomly selects feature values from the benign category to create a new rogue instance. The algorithm then removes duplicated instances that appear in both categories. This procedure is similar to the FrankenCerts approach. It can quickly generate artificial rogue instances, but the generated rogue instances may still be too close to the benign category, which may lead to suboptimal classification performance. 
The anomaly detection approach Negative Selection [Gonzalez et al. 2002], inspired by the human immune system, was the third approach we examined. The approach takes two parameters: the minimum number of mutations $(\mathrm{N})$ and the number of neighboring instances to compare with (M). It starts by generating random values for all machine-learning features. The generated artificial feature set is then compared to the closest $\mathrm{M}$ neighboring instances from the benign category. The generated feature set is acceptable if the distance to the $\frac{M}{2}$ th closest neighbors is at least $\mathrm{N}$. This approach achieves a balanced distance between the benign and rogue instances. However, implementing this approach requires loading the entire benign dataset in the physical memory. The distance comparison can also be time consuming for a large dataset.

\subsection{Selected Approaches}

The design of our generation algorithm for artificial rogue instances needs to meet the following four criteria. First, the generated instances must be sufficiently similar to benign instances but also represent possible variations from them, meaning it is Appropriately Distanced. The design goal is to generate rogue instances with a small distance from the benign instances. The underlying reason is to achieve a balance between the false positives (if too close) and the false negatives (if too distant) of the classification. Second, any generated data must meet requirements for Legitimacy and Validity. All machine-learning features of the artificial rogue instances contain the legitimate and valid values only. Untrusted or invalid certificates can be trivially detected by web browsers and non-browser applications, which are not the focus of this work. The generation algorithms must be Efficient in terms of resource requirements. The algorithm needs to be effective in terms of running time and resource consumption. Since we expect the model training to be conducted periodically with a potentially large set of freshly observed certificates, it is critical that the generation of artificial instances take a reasonable amount of time and does not require enormous computing power. Finally, Consistency is a requirement. The artificial rogue instances should align with the underlying characteristics of the issuing patterns. That is, using the machinelearning features, we should be able to distinguish between different root CA entities.

In order to fulfill these criteria, we designed two separate approaches: Unfocused and Distanced, to generate artificial rogue instances. As cryptographically flawed certificates can trivially be identified as rogue, we did not need to generate actual rogue X.509 certificates. This has the further advantage of not requiring knowledge of the CA's private key (making this work more reproducible). Instead, we determined that synthesizing the features that would be extracted from rogue certificates should they exist is sufficient for detection. Note that the artificial rogue instances for our purposes do not include cryptographic validation but the algorithm as a feature (e.g., MD5) in our design.

The primary idea of the Unfocused approach is simply to use the certificates issued by another root CA entity as rogue instances, since our general approach trains classification models for specific root CA entities. We call the root CA entity for which the model is being trained the focused CA entity and any other root CA entity is unfocused. Although certificates from unfocused CA entities are actually benign, the issuance practices may differ substantially between the root CA entities. For example, when building classification models for Symantec, we can use certificates issued by GoDaddy, Comodo, or Verizon as rogue instances. Note that in the Unfocused classifications, issuer-related fields (e.g., Authority Key ID and issuer DNs) are too strong as indicators and should therefore be excluded in the classification models. This approach helps verify the feasibility of using the selected machine-learning features to uncover 
the issuance practices of different certificate authorities, which matches with our last design criterion-Consistency.

Like other previously discussed approaches, our second approach Distanced generates the artificial rogue instances according to the values in the benign dataset. Specifically, the approach consists of the following steps.

1. Pre-processing. This step is conducted prior to the actual generation of artificial instances. It goes through each benign instance and summarizes the observed values for each machine-learning feature. As the actual generation starts, it is then unnecessary to load the entire benign dataset into the physical memory. This step can help our algorithm meet the third design criterion: effectiveness.

2. Parameter selection. The threshold $N$ is selected to indicate the minimum number of features that need to be mutated from a benign certificate. This step generates a minimum distance between the rogue and benign categories, which satisfies the first design criterion-Appropriately Distanced.

3. Mutating feature selection. The algorithm generates a random integer $m \geq N$ and randomly selects $m$ features to mutate from. This step increases the coverage of possible attacks by randomizing the combination of mutated features.

4. New value generation. For the selected features, the algorithm fills in a random value according to the rules discussed below. This step ensures the validity of the generated instances, which satisfies our second goal of design.

5. Duplicate removal. There is a chance that the generated feature set already exists in the benign category. This step removes these instances from the rogue category to ensure that the two categories are disjoint. This step further protects the minimum distance between the benign and rogue categories.

New feature values of the artificial anomalies are generated according to the five rules. First, for certificate fields with a small finite set of legitimate values the algorithm copies the value from a randomly selected benign instance. This rule applies to standard certificate fields with commonly observed values (for example, the Certificate Version field varies between 0 and 3) and certificate extensions with legitimate values (for instance, the Key Usage extension allows only a fixed set of options).

Second, for certificate fields without a fixed set of legitimate values but can be trivially verified the algorithm also makes a random selection based on observed benign instances. This rule applies to extensions such as CRL Distribution Points or Authority Info Access, where revocation (or other identity-related information) is embedded. Generating entirely random strings for these fields could be easily detected by client-side applications, which is not the focus of this work. Third, for integer features with no limitations, the algorithm randomly generates an integer between the minimum and maximum numbers observed in the benign dataset. This rule applies to features such as the length of the certificate serial number.

Fourth, for all Boolean features, the algorithm randomly generates the value of the feature (True or False). This rule applies to cases such as the Critical label of an extension. Finally, for other features that contain a string value, the algorithm first randomly generates a length of the string, based on the observed benign instances (between the minimum and maximum lengths) and then generates a random string of that length. This rule applies to Authority and Subject Key IDs, and so on.

\section{MODEL GENERATION}

We constructed this system as a back-office data analysis system that observed certificates from the data sources discussed in Section 6. We received daily inputs of certificates from these sources and could train and evaluate models on arbitrary subranges of dates of observations. 


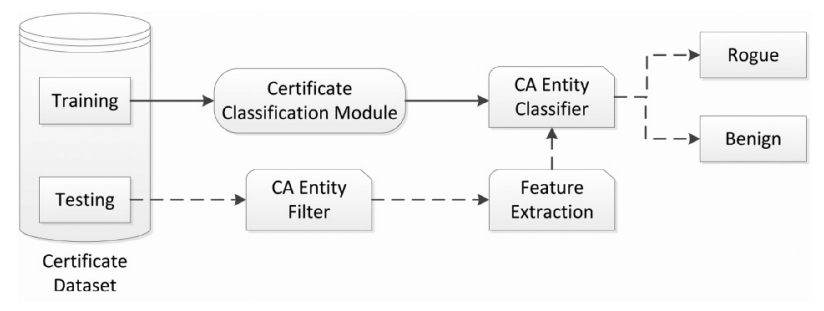

Fig. 5. System deployment.

The machine-learning models built were based on root CA entities, as illustrated in Figure 2. The next practical question is how the machine-learning models are trained so these can be used for detecting rogue certificates. Figure 5 demonstrates the regular workflow of the deployment of our approach. We train the classification models with a number of historical certificate instances observed from a specific root CA entity. Subsequently, for new instances that claim to be from the same root CA entity, the approach extracts the machine-learning features from these new certificates (as with the training datasets) and provides the set of feature values to the corresponding classification model of the root CA entity. The classification model then categorizes the instance into either benign or rogue. As false positives (if the model is too conservative) and false negatives (if it is too aggressive) are a concern, the model is not used as the sole determiner of a certificate's trust but as a filter to eliminate cases with a high probability of being benign. We discuss the potential incorporation of this mechanism in a threat intelligence system in Section 11.1.

One question we had to answer was the length of the training period; that is, the number of days of observations to be included in the training set. While a longer training period can certainly provide more observations of the root CA entity, it may increase the time required for the entire training process. This is an important performance consideration if the models must be regularly retrained to keep pace with a CA's evolving issuance practices. At the same time, there is no guarantee that a longer training period would lead to a higher classification performance. Indeed, the classification performance would only improve when there are new and distinct observations made when a longer training period is considered. With too-short a training period, though, we risk insufficiently capturing the issuance practices in the model. A model with too-high a false-positive rate would not be useful.

By using a separate validation dataset, we examined different lengths of training dataset to achieve the optimal classification performance. We randomly selected a week of data for testing and started with using the certificates observed in the previous week for training. We then increased the number of weeks prior to the testing dataset and recorded the accuracy of the classification. Our results showed that for the top 10 root CA entities, ${ }^{2}$ the constructed DNN classification models achieved the optimal performance when 5-6 weeks of contiguous historical observations were included in the training period. We observed that the accuracy of classification improved monotonically and rapidly until 5 weeks and slightly increased in the 6th week. After that, the performance either remained the same or slightly dropped until the 8th week, when our experiment concluded. We did not continue testing after 8 weeks except for StartCom as we had achieved an acceptable classification performance. Having a shorter training period saves on training time and computing resources and could ease adoption of this technique more broadly. The need for a longer training period for StartCom is further

\footnotetext{
${ }^{2}$ This was ranked by the number of issued certificates in the previous year.
} 
discussed in Section 10. Given that the issuing pattern and the data availability of each root CA entity may change over time, it is important to regularly re-evaluate the optimal training period. CAs with very low issuance rates or very inconsistent issuance patterns may require even longer training periods. But for many, instead of extending the training period, it may be more useful to use a sliding window of the same length where more recent data are incorporated into the model and older data is aged out of it. We consider this on-going maintenance of a model as part of the possible deployment in Section 8.

\section{EVALUATION METHODS}

In terms of measuring the correctness of our classifications, we utilized precision and recall for the "benign" and "rogue" categories, respectively. "Precision" refers to the ratio of True Positives $(T P)$, the correctly classified instances of a certain category, over the number of all instances that are classified as that category: both correctly (True Positives) and incorrectly (False Positives $(F P)$ ). "Recall" indicates the ratio of the True Positives over the number of all instances that are actually in that category: both detected (True Positives) and undetected (False Negatives $(F N)$ ). The overall performance of the trained classifiers is evaluated by Accuracy. This measures the number of instances that were correct, which are both the True Positives and True Negatives, over the entire size of the dataset, which is the number of True Positives, True Negatives $(T N)$, False Positives, and False Negatives. The formulas for these three measurements are as follows:

$$
\text { Precision }=\frac{T P}{T P+F P}, \quad \text { Recall }=\frac{T P}{T P+F N}, \quad \text { Accuracy }=\frac{T P+T N}{T P+T N+F P+F N}
$$

In order to capture a more complete picture of the classification performance, we also calculated the rates of True Positives and False Positives, respectively. These two metrics are calculated as follows:

$$
T P R=\frac{T P}{T P+F N}, \quad F P R=\frac{F P}{F P+T N} .
$$

From the above formulas, it is obvious that the True Positive Rate (TPR) equals the Recall of the same category. To avoid repetition, we list the False Positive Rate (FPR) along with Accuracy, Precision, and Recall in our result tables in the following section.

As a standard practice of performance evaluation for classification models, we also compared the area under the $R O C$ curve for the "rogue" category. The ROC curve is plotted with the True Positive Rate (TPR) along the Y-axis and False Positive Rate (FPR) along the $\mathrm{X}$-axis.

The instances are ranked by their probabilities of belonging to the positive class (in this case, rogue). Each of the probability values can be used as a threshold for splitting the positive and negative classes. A pair of (TPR, FPR) is then calculated for each threshold value and is plotted on the coordinate system. An ROC curve can be plotted by connecting all points within the coordinate system. The ROC curve is a direct illustration of the classification performance. A perfect classifier would demonstrate an ROC curve that is close to the upper left corner, indicating a high true-positive rate with a low false-positive rate. The ideal AUC is therefore 1 (the entire $1 \times 1$ coordinate system). We report the classification results using these evaluation methods in Section 10. 
Table II. Number of Intermediate CA Certs for Each Root CA Entity

\begin{tabular}{ccc}
\hline \multirow{2}{*}{ Root CA Entity } & \multicolumn{2}{c}{ \# of Intermediate Certificates } \\
\cline { 2 - 3 } & $5 / 4 / 2014-6 / 14 / 2014$ & $6 / 15 / 2014-6 / 21 / 2014$ \\
\hline Symantec & 39 & 36 \\
\hline StartCom & 14 & 11 \\
\hline Trustwave & 3 & 3 \\
\hline
\end{tabular}

Table III. Number of Instances Per Category in Each Experiment

\begin{tabular}{ccccccc}
\hline Experiment(s) & \multicolumn{2}{c}{ Unfocused/Distanced I } & \multicolumn{2}{c}{ Distanced II } & \multicolumn{2}{c}{ Distanced III } \\
\hline Dataset & Training & Testing & Training & Testing & Training & Testing \\
\hline Symantec & 2704050 & 468917 & 2811925 & 425074 & 2534751 & 429357 \\
\hline Comodo & 148605 & 27827 & 154508 & 22275 & 131419 & 22750 \\
\hline GoDaddy & 108859 & 18038 & 110160 & 18736 & 108797 & 19280 \\
\hline GlobalSign & 72567 & 12551 & 80683 & 13171 & 81633 & 14067 \\
\hline DigiCert & 1122761 & 245586 & 1467211 & 244696 & 1038336 & 29493 \\
\hline Verizon & 85966 & 13274 & 82192 & 14279 & 82391 & 13902 \\
\hline Entrust & 23162 & 3774 & 23169 & 3869 & 22998 & 4246 \\
\hline StartCom & 11421 & 521 & 12582 & 600 & 12965 & 628 \\
\hline SECOM & 7667 & 1355 & 7422 & 1259 & 6809 & 1026 \\
\hline Trustwave & 5667 & 924 & 5701 & 1037 & 5899 & 1095 \\
\hline
\end{tabular}

\section{PERFORMANCE EVALUATION}

We now report the performance of the classification using first the Unfocused method of providing rogue instances and then using the Distanced method to generate artificial rogue instances, as described in Section 7.

When determining the optimal training periods for the top 10 root CA entities, we discovered that nine classification models achieved their highest performance with 5-6 weeks of training datasets. However, the classification model of StartCom required a significantly longer training period when a 7-day validation set was randomly selected. When comparing the number of certificates in the training datasets of 6 weeks, we observed that only $3 \mathrm{~K}$ certificate chains were under StartCom, while the number of larger root CA entities such as Symantec was 2.7 million. The number of observed StartCom certificates was even smaller than other smaller CAs. For example, Trustwave, which ranked two places smaller than StartCom achieved $5 \mathrm{~K}$ observations during the 6 -week period. Despite other potential reasons related to the classification algorithm, our dataset contained much fewer StartCom certificates encountered by the end users. This inherently led to a longer optimal training period. Additionally, the numbers of intermediate CAs of Symantec, StartCom, and Trustwave are shown in Table II. Intuitively, the training dataset needs to contain a sufficient number of instances per intermediate CA to achieve the optimal classification performance. Symantec issued a large number of certificates through over 30 intermediate CAs, and Trustwave issued $<1 \%$ of the Symantec issued but had only three intermediate CAs. Both of them achieved a rough balance between the number of intermediate CAs and the number of issued certificates. However, this balance was not observed in StartCom, since it has issued fewer certificates from a larger number of intermediate CAs, according to our dataset.

Depending on the volume of issued certificates by each root CA entity, the classification models were given different numbers of training and testing instances, as shown in Table III. Each column illustrates the number of instances in the training or testing category in each of our experiments (Tables IV-VII). Following the procedure described in Section 7, our approach generated roughly the same number of artificial rogue certificates to the benign datasets. 
Table IV. Classification Performance (\%)—Unfocused

\begin{tabular}{ccccccccc}
\hline \multirow{2}{*}{$\begin{array}{c}\text { Root CA } \\
\text { Entity }\end{array}$} & \multirow{2}{*}{ AUC } & \multirow{2}{*}{ Accuracy } & \multicolumn{3}{c}{ Rogue } & \multicolumn{3}{c}{ Benign } \\
\cline { 3 - 8 } & & & Precision & Recall & FPR & Precision & Recall & FPR \\
\hline Symantec & 99.7 & 97.7 & 97.2 & 98.2 & 2.8 & 98.2 & 97.2 & 1.8 \\
\hline Comodo & 97.1 & 91.8 & 91.9 & 91.7 & 8.1 & 91.7 & 91.9 & 8.3 \\
\hline GoDaddy & 97.3 & 92.7 & 94.3 & 90.8 & 5.5 & 91.1 & 94.5 & 9.2 \\
\hline GlobalSign & 97.1 & 90.7 & 88.1 & 94.2 & 12.8 & 93.8 & 87.2 & 5.8 \\
\hline DigiCert & 99.4 & 96.7 & 95.9 & 97.5 & 4.2 & 97.5 & 95.8 & 2.5 \\
\hline Verizon & 98.3 & 94.0 & 92.4 & 96.0 & 8.0 & 95.9 & 92.0 & 4.0 \\
\hline Entrust & 97.5 & 92.2 & 91.3 & 93.4 & 8.9 & 93.3 & 91.1 & 6.6 \\
\hline StartCom & 97.0 & 91.4 & 95.5 & 86.9 & 4.1 & 88.0 & 95.9 & 13.1 \\
\hline SECOM & 98.8 & 94.5 & 93.9 & 95.3 & 6.2 & 95.3 & 93.8 & 4.7 \\
\hline Trustwave & 95.2 & 90.3 & 92.6 & 87.6 & 7.0 & 88.2 & 93.0 & 12.4 \\
\hline
\end{tabular}

Training: 4/3/2014-5/14/2014. Testing: 5/15/2014-5/21/2014.

Table V. Classification Performance (\%)-Distanced I

\begin{tabular}{ccccccccc}
\hline \multirow{2}{*}{$\begin{array}{c}\text { Root CA } \\
\text { Entity }\end{array}$} & \multirow{2}{*}{ AUC } & \multirow{2}{*}{ Accuracy } & \multicolumn{3}{c}{ Rogue } & \multicolumn{3}{c}{ Benign } \\
\cline { 4 - 9 } & & & Precision & Recall & FPR & Precision & Recall & FPR \\
\hline Symantec & 99.7 & 98.0 & 97.7 & 98.3 & 2.3 & 98.3 & 97.7 & 1.7 \\
\hline Comodo & 98.3 & 94.1 & 95.1 & 93.1 & 4.8 & 93.2 & 95.2 & 6.9 \\
\hline GoDaddy & 97.7 & 92.9 & 94.8 & 90.8 & 5.0 & 91.2 & 95.0 & 9.2 \\
\hline GlobalSign & 97.9 & 92.4 & 90.3 & 95.0 & 11.2 & 94.8 & 89.8 & 5.0 \\
\hline DigiCert & 99.8 & 97.1 & 99.6 & 94.7 & 0.4 & 94.9 & 99.6 & 5.3 \\
\hline Verizon & 98.5 & 93.8 & 91.9 & 96.0 & 8.4 & 95.8 & 91.6 & 4.0 \\
\hline Entrust & 98.1 & 94.1 & 93.5 & 94.8 & 6.6 & 94.7 & 93.4 & 5.2 \\
\hline StartCom & 92.1 & 85.8 & 91.5 & 78.9 & 7.3 & 81.5 & 92.7 & 21.1 \\
\hline SECOM & 98.4 & 93.8 & 91.9 & 96.0 & 8.5 & 95.8 & 91.5 & 4.0 \\
\hline Trustwave & 96.7 & 92.4 & 92.2 & 92.6 & 7.8 & 92.6 & 92.2 & 7.4 \\
\hline
\end{tabular}

Training: 4/3/2014-5/14/2014. StartCom Training: 12/3/2013-5/14/2014.

Testing: 5/15/2014-5/21/2014.

Table IV shows the classification performance using the unfocused approach, in which instances in the rogue category are from other root CA entities than the one listed in each row. All top 10 root CA entities have demonstrated strong results using the selected machine-learning features. Both the accuracy and AUC were above 99\%, which is near perfect in this case. These results demonstrate that the underlying issuing patterns are capable of distinguishing one root CA entity from another based on issuance patterns, since identifying information about the CAs (e.g., issuer DNs) was specifically excluded from consideration. Recall that the Unfocused approach was implemented to verify the effectiveness our designed feature set. The actual deployment of the mechanism will be using the Distanced approach, which we introduced in Section 7.

Table $\mathrm{V}$ shows the performance using the artificial rogue instance generation approach with feature values randomly selected from the observed benign instances. The classification results were quite consistent in the top 10 root CA entities in which the AUC values got very close to the perfect cases. The majority of the top root CA entities could achieve an accuracy of $92 \%$. The classification model also demonstrated high performance on the precision and recall rates of the rogue category, with over $90 \%$ in most cases.

Switching the testing window from May to June brought us slightly higher classification results. The AUC rates were all above $94 \%$ for all 10 root CA entities with a high accuracy in these entities, as shown in Table VI. 
Table VI. Classification Performance (\%)—Distanced II

\begin{tabular}{ccccccccc}
\hline \multirow{2}{*}{$\begin{array}{c}\text { Root CA } \\
\text { Entity }\end{array}$} & \multirow{2}{*}{ AUC } & Accuracy & \multicolumn{3}{c}{ Rogue } & \multicolumn{3}{c}{ Benign } \\
\cline { 4 - 9 } & Precision & Recall & FPR & Precision & Recall & FPR \\
\hline Symantec & 99.6 & 97.6 & 98.3 & 96.9 & 1.6 & 96.9 & 98.4 & 3.1 \\
\hline Comodo & 97.4 & 92.4 & 91.7 & 93.2 & 8.4 & 93.1 & 91.6 & 6.8 \\
\hline GoDaddy & 97.2 & 93.0 & 94.3 & 91.6 & 5.5 & 91.9 & 94.5 & 8.4 \\
\hline GlobalSign & 97.7 & 92.7 & 91.6 & 94.1 & 8.6 & 93.9 & 91.4 & 5.9 \\
\hline DigiCert & 100.0 & 99.3 & 98.9 & 99.7 & 1.1 & 99.7 & 98.9 & 0.3 \\
\hline Verizon & 97.9 & 92.8 & 92.0 & 93.7 & 8.1 & 93.6 & 91.9 & 6.3 \\
\hline Entrust & 98.4 & 95.0 & 95.1 & 94.8 & 4.9 & 97.8 & 95.1 & 5.2 \\
\hline StartCom & 95.8 & 90.1 & 91.5 & 88.3 & 8.2 & 88.7 & 91.8 & 11.7 \\
\hline SECOM & 98.1 & 95.1 & 95.8 & 94.3 & 4.1 & 94.4 & 95.9 & 5.7 \\
\hline Trustwave & 94.1 & 91.6 & 94.0 & 88.9 & 5.7 & 89.5 & 94.3 & 11.1 \\
\hline
\end{tabular}

Training: 5/4/2014-6/14/2014. StartCom Training: 1/4/2014-6/14/2014.

Testing: 6/15/2014-6/21/2014.

Table VII. Classification Performance (\%)—Distanced III

\begin{tabular}{ccccccccc}
\hline \multirow{2}{*}{$\begin{array}{c}\text { Root CA } \\
\text { Entity }\end{array}$} & \multirow{2}{*}{ AUC } & \multirow{2}{*}{ Accuracy } & \multicolumn{3}{c}{ Rogue } & \multicolumn{3}{c}{ Benign } \\
\cline { 4 - 8 } & & Precision & Recall & FPR & Precision & Recall & FPR \\
\hline Symantec & 99.7 & 97.7 & 97.2 & 98.2 & 2.8 & 98.2 & 97.2 & 1.8 \\
\hline Comodo & 97.1 & 91.8 & 91.9 & 91.7 & 8.1 & 91.7 & 91.9 & 8.3 \\
\hline GoDaddy & 97.3 & 92.7 & 94.3 & 90.8 & 5.5 & 91.1 & 94.5 & 9.2 \\
\hline GlobalSign & 97.1 & 90.7 & 88.1 & 94.2 & 12.8 & 93.8 & 87.2 & 5.8 \\
\hline DigiCert & 99.4 & 96.7 & 95.9 & 97.5 & 4.2 & 97.5 & 95.8 & 2.5 \\
\hline Verizon & 98.3 & 94.0 & 92.4 & 96.0 & 8.0 & 95.9 & 92.0 & 4.0 \\
\hline Entrust & 97.5 & 92.2 & 91.3 & 93.4 & 8.9 & 93.3 & 91.1 & 6.6 \\
\hline StartCom & 97.0 & 91.4 & 95.5 & 86.9 & 4.1 & 88.0 & 95.9 & 13.1 \\
\hline SECOM & 98.8 & 94.5 & 93.9 & 95.3 & 6.2 & 95.3 & 93.8 & 4.7 \\
\hline Trustwave & 95.2 & 90.3 & 92.6 & 87.6 & 7.0 & 88.2 & 93.0 & 12.4 \\
\hline
\end{tabular}

Training: 6/3/2014-7/14/2014. StartCom Training: 2/3/2014-7/14/2014.

Testing: 7/15/2014-7/21/2014.

Table VII demonstrates the classification performance when the testing dates are set to be between July 15 and July 21 . The AUC rates were and accuracies were even higher than June in most cases, with Symantec reaching 99.7\% in AUC and 97.7\% in accuracy. Smaller root CA entities performed especially well in the classifications.

\section{Detecting a Real-World Attack}

In addition to the performance evaluation using the Unfocused and the Distanced approaches, it is important to examine the classification model against real-world attacks.

As enumerated in the Related Work section, issuance of rogue certificates has occurred in a number of CAs in the recent years. The effects of these incidents can go far beyond a single CA, creating significant damage to the entire PKI ecosystem. Therefore, one natural question would be as follows: Would our machine-learning approach have captured the rogue certificates if it had been deployed prior to those incidents?

All rogue certificates described in the Related Work section were issued with a relatively new NotValidBefore date, only a few days when they are discovered [Hallam-Baker 2011; Fisher 2011; Microsoft 2013; ANSSI 2013]. For these certificates, the number of days since a certificate was issued can become a significant indicator, which has been included in our feature set. Additionally, rogue certificates issued in the TurkTrust incident involved wrongly issued intermediate certificates that allowed further end-entity certificates to be issued. This can be detected by the common chain length, which is also one of the features. This may also apply to the large number of 
rogue CA certificates issued during the DigiNotar incident, to VeriSign, Thawte, DigiCert, and GlobalSign. Further, several CAs tend to issue certificates to their own geographic region, although it is not mandated. This pattern could then be used to identify the rogue certificates of non-governmental sites issued by the French Government CA.

After we had settled on our feature set and evaluation were underway, the compromise of the National Informatics Centre, a subordinate CA of the Indian Controller of Certifying Authorities (India CCA) (a trusted root), was discovered on July 2, 2014. Rogue certificates were issued to several domains belonging to Google and Yahoo!. Because these certificates were issued by a CA present in the trusted root CA list of a major browser, they would be trusted and could be used to make a man-in-the-middle attack.

We trained a model using 2 weeks of certificates observed from the India CCA entity prior to the incident using the method above. We then tested the model against the discovered certificates and discovered that the model classified all of them as rogue. Further analysis showed that the fraudulent certificates differed from the normal observations in the following features.

1. Subject Country. All benign certificates in the training set were issued to a subject in India, but the rogue certificates were issued to U.S. entities.

2. Subject Alternative Name. Most of the benign certificates only had one listed entry, but the fraudulent examples had a large number of entries.

3. (Not Valid After-Not Valid Before). Most of the benign instances had a validity period of at least 700 days, but the rogue certificates were only valid for a year.

4. (Observation-Not Valid Before). When the certificate was first seen, its Not Valid Before date was just a few days before. Most of the benign instances seen in our data sets had much earlier issuance dates.

Note that the rules listed above are only one possible combination of feature values that can be used to distinguish the benign and rogue India CCA certificates. For this particular compromise, the differences between normal certificates and the rogue cases were sufficiently simple-a rule-based approach would also have detected them. However, this would necessitate the a priori construction of such rules for each CA, which is infeasible for anyone other than the CA itself. Indeed, the point of using a machine-learning approach in this case is to learn a CA's issuance behavior without cooperation from it. The DNN model relied on a more complex set of features to make the decision. The DNN models are capable of identifying more complex relationships between the features, which can be useful to detect more sophisticated designed rogue certificates in the future, particularly in cases where the differences are not sufficiently obvious to be noticed by humans. The serendipity of this attack occurring during our research allowed us an opportunity to apply our technique to an attack that was not known when designing our approach. It remains an open question whether or not this technique will be able to detect more sophisticated future attacks. As mentioned earlier, applying this technique to known attacks on which the technique was developed would not be indicative of this, but that this technique surfaced the rogue certificates from this attack for further examination is encouraging.

\section{DISCUSSION AND FUTURE WORK}

\subsection{Possible Deployments}

Our proposed machine-learning mechanism can be deployed by any interested organizations or corporations, including the certificate authorities themselves. Currently, the system is deployed in test mode at a major browser manufacturer. The system was 
deployed in this test mode when the India CCA rogue certificates were identified as "Rogue."

Major operating system and browser manufacturers can use their previously observed certificates to generate machine-learning models for root CA entities. These corporations/organizations often operate individual root CA programs and therefore interact directly with the trusted CAs. Additionally, any interested online entities with certificate telemetry can deploy our approach to identify suspicious certificates from the trusted CAs. As CAs log more certificates with Certificate Transparency, it becomes another potential source of certificates for analysis.

This method can then be integrated into the threat intelligence and analysis system at such an organization. These systems use a variety of automated techniques to filter down signals and telemetry to a quantity manageable by human threat analysts, who then decide what warrants further action. In the same way, this model can be integrated into such a process, initially with a conservative stance that is more likely to generate false positives rather than false negatives, so potentially malicious cases are surfaced to analysts. This process naturally leads into a feedback loop where the automated process is further refined to provide better filtered and therefore more useful data to the analysts. This will allow us to tune the model to be more aggressive with confidence that relevant threat indicators are not being filtered. In part, this mechanism exchanges one part-time trained security analyst for an immediate decrease in the window of vulnerability, without the requirements for changing the infrastructure. Since trained personnel are needed to staff notaries and centralized services, the requirement for some staff time is arguably a part of all proposals.

Certificate authorities often do not keep aggregate records of all certificates issued from their hierarchy, particularly when the intermediate CA is operated by another organization. Instead, they keep a record of intermediate CAs issued by the root and those intermediate CAs are responsible for keeping records of their issued certificates. Our approach can therefore help the higher-level CAs (on the trusted chain) observe and detect any rogue certificates issued by lower-level CAs, if the trusted chain is of a length greater than 2 . With the list of issued certificates, our approach can still serve as a mechanism to detect possible rogue certificates not captured by internal audit.

\subsection{Attacker Responses}

Once our approach is deployed, adversaries may adjust their strategy in order to circumvent the deployed system. Attacks on machine-learning models have attracted more attention in the security community, as these mechanisms are being used in an increasing number of security domains, such as the detection of phishing [Basnet et al. 2008; Xiang et al. 2011; Rosiello et al. 2007; Whittaker et al. 2010], malware [Bailey et al. 2007], and botnets [Gu et al. 2008].

In this subsection, we analyze two typical adversarial attacks: poisoning and evasion, and discuss the robustness of our approach against these attacks.

We assume, out of necessity, that all of the data in our training set is benign, and from that we synthesize rogue data. We also recognize that models must be regularly retrained based on recent observations in order to keep them current with respect to a root CA entity's issuance practices, which change over time. This does open the possibility that an adversary who has compromised a CA and is aware of this approach might attempt to attack the statistical strength of the data. This could be done by poisoning the model by introducing subtle variations in order to "move the needle" towards their ultimate attack goal in the hopes that, when the rogue certificate created for the true attack was made, the model would classify the rogue certificate as benign. This is a danger for any regularly retrained machine-learning model. Given that the number of new certificates observed every day number in the thousands to tens of thousands, we 
believe the sheer number of aberrant instances would itself stand out as an aberration. Assuming these instances were in addition to all of the benign certificates issued by a CA, given that we propose the presence of human analysts in any CA compromise detection system, the conspicuous increase would itself be an indicator of possible compromise. If instead the attacker was able to change the issuance practices of the root CA entity in some way that made a later attack easier, then that might be noticed by an earlier model before a new model was trained.

The second type of adversarial machine-learning attack is evasion. Specifically, with the knowledge of the classification model and/or the feature set, the adversary could manipulate the values in certain certificate fields so the new rogue certificate would look similar to the benign instances. While we have assumed a strong adversary, the certificates themselves are highly constrained in terms of semantics (i.e., the adversary cannot circumvent the system by simply copying the field values from a benign certificate to generate a rogue one). Because of the strictly defined certificate structure, changes to certain certificate fields can sometimes be difficult to make. The changes in certificates are also highly observable compared to other security domains. Almost all previously observed rogue certificates deviated from the CA's standard issuance practices. In addition, our generated machine-learning models are re-trained at a regular basis and may include an adjustment of machine-learning features, depending on the evolution of the rogue certificates in the future.

Consider the validity-related fields. A strong adversary may be able to issue backdated certificates, although we have not observed this in known certificate compromise incidents. We argue that these backdated certificates, once issued, can be identified by a joint effort of our approach (since NotValidBefore is not the only strong feature) and existing organizations such as the $\mathrm{CA} /$ Browser Forum and its processes.

If we consider phishing detection as an analogous research area, then the AntiPhishing Working Group is an example of a dedicated organization to address this particular type of attack. Multiple entities exist to generate the Black Hole List against spammers. We argue that similar investments against rogue certificates would be likely to rise in the case of an advanced persistent threat. Considering that normal phishing websites have an average lifetime of less than 3 days before being blacklisted [Moore and Clayton 2007], if the rogue certificates were backdated too much with equivalent telemetry to the phishing case, then we can expect that such an attack would be identified at a comparable rate.

\subsection{Limitations and Future Work}

Machine-learning classifiers by their very definition will turn up a certain number of false positives. Indeed, a classifier which has a particularly low or zero rate of false positives instead likely has an unacceptably high false-negative rate. In this case, it is better to examine a legitimate certificate than to accept a rogue certificate. The high accuracy rates of the classifiers we have trained and tested so far suggest a possible deployment configuration: narrowing down the new certificates seen every day down to a number that could be reasonably triaged by a human operator for possible further investigation, much as is currently done with a number of intrusion detection systems. Over time, these analysts could participate in a feedback loop to further refine construction of the model to provide even better results. We described the proposed procedure in Section 8 .

By having only benign data as a basis for training, we rely on the assumption that rogue certificates will always differ from benign ones in some significant way that will be surfaced by a machine-learning model. Our practical experience with the India CCA is encouraging but we must observe that this is a small root CA entity that historically issued certificates only to a certain type of site (primarily government) in 
a particular geography (India). Although we did observe variations from the normal issuance practices of this CA, we must be cautious not to underestimate the impact of the different geographies of the benign and rogue certificates in this case. It remains an open question if these deviations will be sufficient to detect future rogue certificates from a CA that issues certificates more broadly, and we will have to analyze this if and when future compromises occur.

We will perform further evaluation of our mechanism without the connection-related features for the top 10 root CA entities, although the classification performance with solely the certificate fields have already been tested in the India CCA experiment. This suggests that while additional metadata are helpful, the technique still performs well with features drawn only from the publicly observable certificates themselves.

In future work, we will also examine the possibility of providing a probabilistic estimate to end users with respect to likelihood of a rogue certificates. This work requires expanding our research into the usability, risk communication, and warnings domain. The current deployment identifies rogue CAs that are then revoked, leaving both the PKI and the user experience unchanged. While end user experience is beyond the scope of this article, we recognize it as an important domain for future research.

As part of this research, we have made our certificates compilation available to the research community. Interested researchers can request access through the PREDICT project, which recently renamed IMPACT. ${ }^{3}$

Our machine-learning-based fraudulent certificate detection mechanism is an effort to quickly identify attacks in the existing PKI ecosystem. Reasonable hope of deployment means that the attacker response scenarios become an area of future work. Thus, our research includes perturbations of the artificial instances to determine if particular patterns could weaken the classification results. A systematic redesign of PKI that could completely avoid fraudulent certificates from being issued is a more complex question. Such a redesign may require fundamental changes to the current certificate issuance practices and would require collaborative action of many of the participating parties in the PKI ecosystem. It is beyond the horizon of this work.

\section{CONCLUSION}

The current PKI ecosystem relies on the trustworthiness of certificate authorities. Because of this fundamental assumption, rogue certificates are a threat. Any certificate authority may issue certificates to any website, and all certificates from a trusted CA are trusted with no discernible difference to the end user. However, CAs have been compromised by adversaries, causing technically valid and trusted but rogue certificates to be issued to those adversaries. In this work, we proposed an effective machine-learning-based mechanism to detect potentially rogue certificates and show a promising result with respect to the India CCA compromise incident. We described the deployment under which this compromise was identified as a practical use scenario. We illustrated that the detection of rogue certificates case matches the criteria for a security domain where machine learning is applicable. We enumerated the features relevant for machine learning and identified the algorithm with the greatest accuracy.

\section{REFERENCES}

Bernhard Amann, Robin Sommer, Matthias Vallentin, and Seth Hall. 2013. No attack necessary: The surprising dynamics of SSL trust relationships. In Proc. of ACSAC'13. ACM, 179-188.

Bernhard Amann, Matthias Vallentin, Seth Hall, and Robin Sommer. 2012. Extracting Certificates from Live Traffic: A Near Real-Time SSL Notary Service. Technical Report. TR-12-014, ICSI.

\footnotetext{
$\overline{{ }^{3} \mathrm{https}: / / w w w . p c h . n e t / r e s o u r c e s / C e r t i f i c a t e s / . ~}$
} 
ANSSI. 2013. Revocation of an IGC/A branch. http://www.ssi.gouv.fr/en/the-anssi/events/revocation-of-anigc-a-branch-808.html. (Dec 2013).

Michael Bailey, Jon Oberheide, Jon Andersen, Z. Morley Mao, Farnam Jahanian, and Jose Nazario. 2007. Automated classification and analysis of internet malware. In Recent Advances in Intrusion Detection. Springer, 178-197.

Ram Basnet, Srinivas Mukkamala, and Andrew H. Sung. 2008. Detection of phishing attacks: A machine learning approach. In Soft Computing Applications in Industry, Bhanu Prasad (Ed.). Studies in Fuzziness and Soft Computing, Vol. 226. Springer, Berlin, 373-383.

Lujo Bauer, Scott Garriss, and Michael Reiter. 2011. Detecting and resolving policy misconfigurations in access-control systems. ACM Trans. Inform. Syst. Secur. 14, 1 (2011), 2.

Yoshua Bengio. 2009. Learning deep architectures for AI. Found. Trends Mach. Learn. 2, 1 (2009), 1-127.

Léon Bottou. 1991. Stochastic gradient learning in neural networks. Proc. Neuro-Nimes 91, 8 (1991).

Leo Breiman. 2001. Random forests. Mach. Learn. 45, 1 (2001), 5-32.

Chad Brubaker, Suman Jana, Baishakhi Ray, Sarfraz Khurshid, and Vitaly Shmatikov. 2014. Using Frankencerts for automated adversarial testing of certificate validation in SSL/TLS implementations. In Proc. of SP'14. IEEE Computer Society, 114-129.

Nitesh V. Chawla. 2005. Data mining for imbalanced datasets: An overview. In Data Mining and Knowledge Discovery Handbook. Springer, 853-867.

Nitesh V. Chawla, Kevin W. Bowyer, Lawrence O. Hall, and W. Philip Kegelmeyer. 2002. SMOTE: Synthetic minority over-sampling technique. J. Artif. Intell. Res. 16, 1 (Jun. 2002), 321-357.

Corinna Cortes and Vladimir Vapnik. 1995. Support-vector networks. Mach. Learn. 20,3 (1995), $273-297$.

Sevtap Duman, Kaan Onarlioglu, Ali Osman Ulusoy, William Robertson, Engin Kirda, Erik-Oliver Blass, Travis Mayberry, Guevara Noubir, Kaan Onarlioglu, Michael Weissbacher, and others. 2014. TrueClick: Automatically distinguishing trick banners from genuine download links. In Proc. of ACSAC'14. ACM, 456-465.

Zakir Durumeric, James Kasten, Michael Bailey, and J. Alex Halderman. 2013. Analysis of the HTTPS certificate ecosystem. In Proc. of IMC'13. ACM, 291-304.

Kevin P. Dyer, Scott E. Coull, Thomas Ristenpart, and Thomas Shrimpton. 2012. Peek-a-boo, i still see you: Why efficient traffic analysis countermeasures fail. In Proc. of SP'12. IEEE, 332-346.

Dumitru Erhan, Yoshua Bengio, Aaron Courville, Pierre-Antoine Manzagol, Pascal Vincent, and Samy Bengio. 2010. Why does unsupervised pre-training help deep learning? J. Mach. Learn. Res. 11 (2010), $625-660$.

Nicolas Falliere, Liam O. Murchu, and Eric Chien. 2011. W32. stuxnet dossier. White Paper, Symantec Corp., Security Response (2011).

Wei Fan, Matthew Miller, Sal Stolfo, Wenke Lee, and Phil Chan. 2004. Using artificial anomalies to detect unknown and known network intrusions. Knowl. Inform. Syst. 6, 5 (2004), 507-527.

Dennis Fisher. 2011. DigiNotar Says Its CA Infrastructure Was Compromised. Retrieved from https:// threatpost.com/diginotar-says-its-ca-infrastructure-was-compromised-083011/75594/.

CA/Browser Forum. 2015. Baseline Requirements Certificate Policy for the Issuance and Management of Publicly-Trusted Certificates. Retrieved from https://cabforum.org/wp-content/uploads/CAB-Forum-BR1.3.0.pdf.

Laura Fumanelli, Marco Ajelli, Piero Manfredi, Alessandro Vespignani, and Stefano Merler. 2012. Inferring the structure of social contacts from demographic data in the analysis of infectious diseases spread. PLoS Comput. Biol. 8, 9 (Sep. 2012), e1002673.

Sujata Garera, Niels Provos, Monica Chew, and Aviel D. Rubin. 2007. A framework for detection and measurement of phishing attacks. In Proc. of WORM'07. ACM, 1-8.

Fabio Gonzalez, Dipankar Dasgupta, and Robert Kozma. 2002. Combining negative selection and classification techniques for anomaly detection. In Proc. of CEC'02, Vol. 1. IEEE, 705-710.

Guofei Gu, Roberto Perdisci, Junjie Zhang, Wenke Lee, and others. 2008. BotMiner: Clustering analysis of network traffic for protocol-and structure-independent botnet detection. In Proc. of USENIX Security'08. USENIX, 139-154.

Phillip Hallam-Baker. 2011. Comodo SSL Affiliate The Recent RA Compromise. Retrieved from https://blogs. comodo.com/uncategorized/the-recent-ra-compromise/.

Ling Huang, Anthony D. Joseph, Blaine Nelson, Benjamin I. P. Rubinstein, and J. D. Tygar. 2011. Adversarial machine learning. In Proc. of AISec'11. ACM, 43-58.

Lin Shung Huang, Alex Rice, Erling Ellingsen, and Collin Jackson. 2014. Analyzing forged SSL certificates in the wild. In Proc. of SP'14. IEEE Computer Society, 83-97. 
IETF. 2008. Internet X.509 Public Key Infrastructure Certificate and Certificate Revocation List (CRL) Profile. http://tools.ietf.org/html/rfc5280. (May 2008).

Josh Karlin, Stephanie Forrest, and Jennifer Rexford. 2006. Pretty good BGP: Improving BGP by cautiously adopting routes. In Proc. of ICNP'06. IEEE, 290-299.

Timothy Kelley and L. Jean Camp. 2012. Online promiscuity: Prophylactic patching and the spread of computer transmitted infections. In Proc. of WEIS'12. Springer.

Richard L. Barnes. 2011. DANE: Taking TLS authentication to the next level using DNSSEC. IETF J. (Oct. 2011).

Jon Larimer and Kenny Root. 2012. Security and Privacy in Android Apps. Retrieved from https://developers. google.com/events/io/2012/sessions/gooio2012/107/.

Ben Laurie, Adam Langley, and Emilia Kasper. 2013. RFC 6962: Certificate transparency. http://www. rfceditor.org/info/rfc6962.

Saskia Le Cessie and Johannes C. Van Houwelingen. 1992. Ridge estimators in logistic regression. Appl. Stat. 41, 1 (1992), 191-201.

Tie-Yan Liu, Yiming Yang, Hao Wan, Hua-Jun Zeng, Zheng Chen, and Wei-Ying Ma. 2005. Support vector machines classification with a very large-scale taxonomy. ACM SIGKDD Explor. Newslett. 7, 1 (2005), 36-43.

Justin Ma, Lawrence K. Saul, Stefan Savage, and Geoffrey M. Voelker. 2009. Identifying suspicious URLs: An application of large-scale online learning. In Proc. of ICML'09. ACM, 681-688.

Michelle L. Mazurek, Saranga Komanduri, Timothy Vidas, Lujo Bauer, Nicolas Christin, Lorrie Faith Cranor, Patrick Gage Kelley, Richard Shay, and Blase Ur. 2013. Measuring password guessability for an entire university. In Proc. of CCS'13. ACM, 173-186.

Ralph C. Merkle. 1988. A digital signature based on a conventional encryption function. In Proc. of CRYPTO'87. Springer, 369-378.

Microsoft. 2013. Microsoft Security Advisory 2798897: Fraudulent Digital Certificates Could Allow Spoofing. Retrieved from https://technet.microsoft.com/library/security/2798897.

Microsoft. 2014a. Manage Trusted Root Certificates. Retrieved from https://technet.microsoft.com/en-us/ library/cc754841.aspx.

Microsoft. 2014b. Microsoft Security Advisory 2982792: Improperly Issued Digital Certificates Could Allow Spoofing. https://technet.microsoft.com/en-us/library/security/2982792.aspx. (Jul 2014).

Mishari Al Mishari, Emiliano De Cristofaro, Karim El Defrawy, and Gene Tsudik. 2009. Harvesting SSL certificate data to identify web-fraud. arXiv preprint arXiv:0909.3688 (Sep 2009).

Tyler Moore and Richard Clayton. 2007. Examining the impact of website take-down on phishing. In Proc. of APWG eCrime'07. APWG, 1-13.

Mozilla. 2015. CA:AddRootToFirefox: Installing Certificates into Firefox. Retrieved from https://wiki. mozilla.org/CA:AddRootToFirefox.

Angelo P. E. Rosiello, E. Kirda, C. Kruegel, and F. Ferrandi. 2007. A layout-similarity-based approach for detecting phishing pages. In Proc. of SecureComm'07. Springer, 454-463.

A. H. Schistad Solberg and R. Solberg. 1996. A large-scale evaluation of features for automatic detection of oil spills in ERS SAR images. In Proc. of IGARSS'96, Vol. 3. 1484-1486.

Robin Sommer and Vern Paxson. 2010. Outside the closed world: On using machine learning for network intrusion detection. In Proc. of SP'10. IEEE, 305-316.

Andreas P. Streich, Mario Frank, David Basin, and Joachim M. Buhmann. 2009. Multi-assignment clustering for Boolean data. In Proc. of ICML'09. ACM, 969-976.

James Theiler and D. Michael Cai. 2003. Resampling approach for anomaly detection in multispectral images. In Proc. of SPIE Aerosense'03. International Society for Optics and Photonics, 230-240.

Adam Toon. 2012. Models as Make-Believe: Imagination, Fiction and Scientific Representation. Palgrave Macmillan.

Tor. 2011. The DigiNotar Debacle, and What You Should Do About It. Retrieved from https://blog.torproject. org/blog/diginotar-debacle-and-what-you-should-do-about-it.

Gang Wang, Tianyi Wang, Haitao Zheng, and Ben Y. Zhao. 2014. Man vs. machine: Practical adversarial detection of malicious crowdsourcing workers. In Proc. of USENIX Security'14. USENIX, 239-254.

Michael Weisberg. 2013. Simulation and Similarity: Using Models to Understand the World. Oxford University Press.

Dan Wendlandt, David G. Andersen, and Adrian Perrig. 2008. Perspectives: Improving SSH-style host authentication with multi-path probing. In Proc. of USENIX'08, Vol. 200. USENIX, 321-334. 
Colin Whittaker, Brian Ryner, and Marria Nazif. 2010. Large-scale automatic classification of phishing pages. In Proc. of NDSS'10. ISOC.

Guang Xiang, Jason Hong, Carolyn P. Rose, and Lorrie Cranor. 2011. CANTINA+: A feature-rich machine learning framework for detecting phishing web sites. ACM Trans. Inf. Syst. Secur. 14, 2 (Sep 2011), 21:1-21:28.

Received February 2015; revised April 2016; accepted July 2016 